\title{
NIR spectral heritability: a promising tool for wood breeders
}

\author{
PRG Hein ${ }^{1}$, M Denis², PF Trugilho 3 , JT Lima ${ }^{4}$, AM Rosado ${ }^{5}$ and G Chaix
}

\section{Introduction}

As a fast-growing and short-rotation source of wood, Eucalyptus plantations are the basis for several industries, such as paper and steel producers. Forest sectors demand phenotypes exploitable for a wide range of processing needs according for different end-uses, allowing effective response to the rapidly changing market, technological, and natural environments (Verryn 2008). Hence, the determination of the genetic factors contributing to quantitative trait variation of wood properties is essential for tree breeders. This study focuses on verifying in which extent the Near Infrared (NIR) spectra variations recorded on woods from Eucalyptus are under genetic and environmental controls. NIR spectroscopy is a fast, non-destructive technique based on vibrational spectroscopy which expresses the interaction between radiation and the material.

\section{Material and methods}

One hundred fifty (150) discs at $25 \%$ of tree height of 6-year old Eucalyptus grandis $\mathrm{x}$ E. urophylla hybrids coming from clonal tests established in Brazil on three nearest sites $\left(19^{\circ} 17^{\prime} \mathrm{S}, 42^{\circ} 23^{\prime} \mathrm{W}\right.$, alt $\left.230-500 \mathrm{~m}\right)$ were used in this study. NIR spectra were recorded using a spectrophotometer (model Vector 22/N, Bruker Optik $\mathrm{GmbH}$, Ettlingen, Germany) along radius (outer, intermediate and outer) of radial face of the wood discs. The samples were stabilized at $12 \%$ of moisture content beforehand. Second derivatives (25-point filter and a third order polynomial) were applied on the spectra to enhance the quality of the information. NIR spectra were recorded from 9,000 to $3,500 \mathrm{~cm}^{-1}$ totalizing 1,400 absorption values. Each NIR spectrum was reduced along its wavenumber by a reduction factor of 5 producing a NIR spectrum containing 280 absorption values. Each wavelength was analyzed

\footnotetext{
${ }^{1}$ Ciência e Tecnologia da Madeira - Departamento de Ciências Florestais, Universidade Federal de Lavras - campus universitário Lavras, Minas Gerais, Brazil, CEP 37200-000, email: phein1980@gmail.com

${ }^{2}$ CIRAD - Genetic improvement and adaptation of mediterranean and tropical plants, International campus of Baillarguet TA A-108/C - 34398 Montpellier Cedex 5 France

${ }^{3}$ Ciência e Tecnologia da Madeira - Departamento de Ciências Florestais, Universidade Federal de Lavras - campus universitário Lavras, Minas Gerais, Brazil, CEP 37200-000, email: trugilho@dcf.ufla.br

${ }^{4}$ Ciência e Tecnologia da Madeira - Departamento de Ciências Florestais, Universidade Federal de Lavras - campus universitário Lavras, Minas Gerais, Brazil, CEP 37200-000, email: jtlima@dcf.ufla.br

${ }^{5}$ Celulose Nipo-Brasileira S.A, Rodovia BR 381, Km 172 , Belo Oriente, Minas Gerais, Brazil, CEP 35196-000

${ }^{6}$ CIRAD - Genetic improvement and adaptation of mediterranean and tropical plants, 73 rue Jean-François Breton TA B-40/ 16, 34398 Montpellier, Cedex 5, France
} 
independently (univariate analysis) to estimate the variance components by using an individual mixed linear model as $y=\mu+$ Clone + Site + ClonexSite $+\varepsilon$ where ì is the mean value, Clone is the random effect, Site is the fixed effect, ClonexSite is random interaction effect and $\varepsilon$ is the residual. The variances associated to random and fixed effects were estimated by restricted maximum likelihood (REML) analysis by using computer routines written in the $R$ statistical programming language $(R$ Development Core Team 2008). As the variances are assumed to be independent, the total phenotypic variance was calculated as $\sigma_{p}^{2}=\sigma_{g}^{2}+\sigma_{g x e}^{2}+\sigma_{e^{\prime}}^{2}$ where $\sigma_{p}^{2}$ is the phenotypic variance, $\sigma_{\mathrm{g}}^{2}$ is the clonal variance, $\sigma_{\mathrm{gxe}}^{2}$ is the clone by site interaction variance and $\sigma_{e}^{2}$ is the environmental (error) variance. The broad-sense heritabilities were estimated as $H^{2}=\sigma_{g}^{2} /\left(\sigma_{g}^{2}+\sigma_{g x e}^{2}+\sigma^{2}{ }_{e}\right)$. As the NIR spectrum consisted of 280 wavenumbers, the same number of broad-sense NIR spectral heritabilities was performed.

\section{Results and discussion}

NIR spectra contain a lot of information about wood, this complex threedimensional biopolymer material composed of an interconnected network of cellulose, hemicelluloses, lignins and extractives. Considering a NIR spectrum as a source of information concerning many wood traits, genetic parameters were calculated from them.

The broad-sense NIR spectral heritabilities and the radial variation are

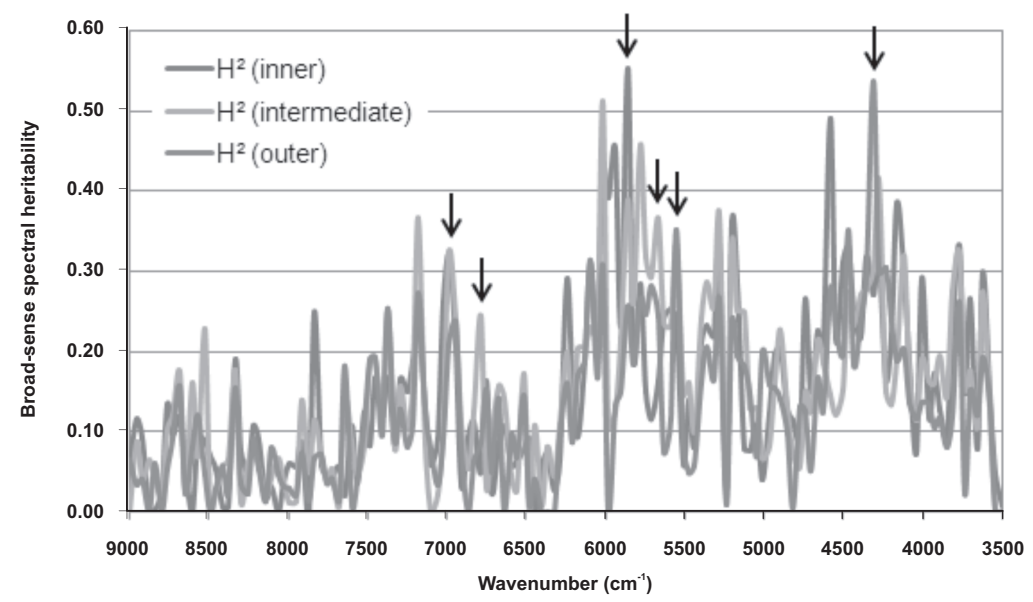

Figure 1. Radial variation of NIR spectral broad-sense heritability estimates. 
presented by colors (Figure 1). The analysis of the NIR spectra heritabilities by wavenumber plot is useful to investigate the underlying relationships that have made the estimation of genetic parameters possible by NIR spectroscopy. The assignments of absorption bands are useful to identify which wood components present higher broad-sense heritabilities from NIR spectroscopic data. It helps to understand how NIR spectroscopy can evaluate genetic control over wood traits.

Some ranges of the NIR spectra presented heritabilities greater than $50 \%$. This means that NIR spectra are able to capture the potential genetic of some chemical components and wood traits indirectly. The bands normally associated with cellulosic or lignin type biomolecules may be observed in the NIR spectra of wood. For comparative purpose, the heritability of the wood density estimated by NIRbased models at the same points linearly increased from 0.24 to 0.49 towards the cambium.

The indicated bands yielded high NIR spectra heritabilities. These bands are normally associated to cellulose and lignin contents. According to Workman and Weyer (2007) the band around $6,831 \mathrm{~cm}^{-1}$ is associated to $\mathrm{O}-\mathrm{H}$ polymeric (2íO-H) and is assigned to cellulose type I. The NIR spectral heritabilities also were high around 5,669 $\mathrm{cm}^{-1}$ and this band is associated to functional grouping $\mathrm{CH}_{2}$ : stretching (2í) being related to cellulose content. The bands around 5,553 $\mathrm{cm}^{-1}$ and at 4,313 $\mathrm{cm}^{-1}$ are also related to the cellulose content of the wood. Lignin is representative of aromatic natural product compounds. The band around $7,180 \mathrm{~cm}^{-1}$ is associated to $\mathrm{C}-\mathrm{H}\left(2 \mathrm{iCH}_{2}\right.$ and $\left.\mathrm{aCH}_{2}\right)$ and is interrelated to lignin content. The band around 5,940 $\mathrm{cm}^{-1}$ belongs to functional grouping $\mathrm{C}-\mathrm{H}(2 \mathrm{i})$, ArcC- $\mathrm{H}$ : $\mathrm{C}-\mathrm{H}$ aromatic associated to $\mathrm{C}-$ $\mathrm{H}$, also related to lignin content. The NIR spectral heritability estimates also were high around 4,585 $\mathrm{cm}^{-1}$ and this band is associated to $\mathrm{C}-\mathrm{H}$ stretching and $\mathrm{C}=\mathrm{O}$ combination related to lignin content of wood.

\section{Concluding remarks}

In moisture content controlled situation, the variations in NIR spectra are related to variation of chemical traits as lignin, and cellulose, and hemicelluloses, and extractives contents. It appears that variations in the indicated wavenumbers (which are related to chemical components of wood) can be attributed to genetic effects.

As NIR spectra is a countless source of information concerning wood traits, the analysis of genetic parameters from NIR spectra of wood appears to be an efficient and promising way to indirectly evaluate the genetic control over many wood traits at once and the efficiency of the design. 
254 - Oral Session

\section{References}

R Development Core Team. 2008. R: A Language and Environment for Statistical Computing. Vienna, Austria: R Foundation for Statistical Computing. ISBN 3900051-07-0. http://www.r-project.org.

Verryn SD (2008) Breeding for wood quality - a perspective for the future. New Zealand Journal of Forestry Science 38(1): 5-13

Workman J and Weywer L (2007) Practical guide to interpretive near infrared spectroscopy. Boca Raton: CRC Press. 332p. 
IUFRO Working Group 2.08.03

IMPROVEMENT AND CULTURE OF EUCALYPTS

\section{PROCEEDINGS}

PORTO SEGURO, BAHIA, BRAZIL

$14^{\text {th }}$ to $18^{\text {th }} \mid$ NOVEMBER $\mid 2011$ 